\title{
Quantitative Morphology of Full Thickness Rotator Cuff Tears
}

\author{
JASON D. WENING, RONALD F. HOLLIS, RICHARD E. HUGHES, ${ }^{*}$ aND JOHN E. KUHN \\ University of Michigan, Orthopaedic Research Lab, Ann Arbor, Michigan
}

\begin{abstract}
The occurrence of full thickness rotator cuff tears (RCTs) at time of death, the size and distribution of those tears among the tendons of the rotator cuff, and the anterior to posterior and medial to lateral dimensions of the RCTs were determined. A subset of 57 shoulders from a group of 414 were found to have full thickness rotator cuff tears. The three-dimensional coordinates of specific bony landmarks and points defining the circumference of the RCT were digitized using a Flock of Birds DC electromagnetic tracking device. Bony landmarks were used to determine the separation between adjacent tendons of the rotator cuff. Points describing the circumference of the RCT were used to calculate the area of the tear. The majority of tears occurred in the supraspinatus tendon alone (25), or in both the supraspinatus and infraspinatus tendons (22). The areas of the RCTs ranged from $0.07 \mathrm{~cm}^{2}$ to $19.17 \mathrm{~cm}^{2}$ with an average of $4.43 \mathrm{~cm}^{2}$. The mean anterior to posterior length was $1.95 \mathrm{~cm}$. The mean medial to lateral length was $1.98 \mathrm{~cm}$. Clin. Anat. 15:18-22, 2002. ๑ 2002 Wiley-Liss, Inc.
\end{abstract}

Key words: rotator cuff tendons; prevalence; supraspinatus; infraspinatus; subscapularis

\section{INTRODUCTION}

Several studies have reported the prevalence of full thickness rotator cuff tears (RCTs) in the general population at the time of death (Bigliani et al., 1986; Hijioka et al., 1993; Keyes, 1933; Lehman et al., 1995; Ogata and Uhthoff, 1990; Wilson and Duff, 1943; Yamanaka et al., 1983; Zuckerman et al., 1992). A few authors have reported qualitative assessments of the distribution of full thickness tears among the rotator cuff tendons (Itoi et al., 1995). Others have reported qualitative assessments of RCT size using the Bateman scale (Tempelhof et al., 1999). Sher et al. (1995) reported quantitative measures of RCTs in asymptomatic individuals based on MR images. Although grading tears according to small, medium, large, and massive sizes is clinically useful, new advances in computational modeling of the rotator cuff (Chang et al., 2000; Hughes and An, 1997; Hughes et al., 1999) create a need for more accurate quantitative measures of tear size and percentage of each tendon involved in a tear. Shoulder strength in normal subjects has been predicted from geometric and physiological data (Hughes et al., 1999), but prediction of strength deficits in a population of shoulders with RCTs requires information about the statistical distribution of cuff tear morphology. Obtaining the most accurate measurements requires digital collection of data from carefully dissected specimens and computational estimation of the area of each tear.

The purpose of this study was to accurately determine the area of full thickness RCTs and to determine the portion of the tear included in each of the rotator cuff tendons. Descriptive statistics for the prevalence of full thickness RCTs at the time of death based on age, sex, and side of body are presented. The dimensions of tears in the supraspinatus tendon were measured in the anterior-to-posterior and medial-tolateral directions.

\section{MATERIALS AND METHODS}

Between May, 1994, and March, 1999, a convenient sample of 414 cadaver shoulders were collected from a population of men and women who donated their bodies to the University of Michigan Anatomical Do-

*Correspondence to: Richard Hughes, Ph.D., University of Michigan, Orthopaedic Research Lab, 400 North Ingalls Building G-161, Ann Arbor, MI 48109-0486.

Received 8 May 2000; Revised 6 October 2000 


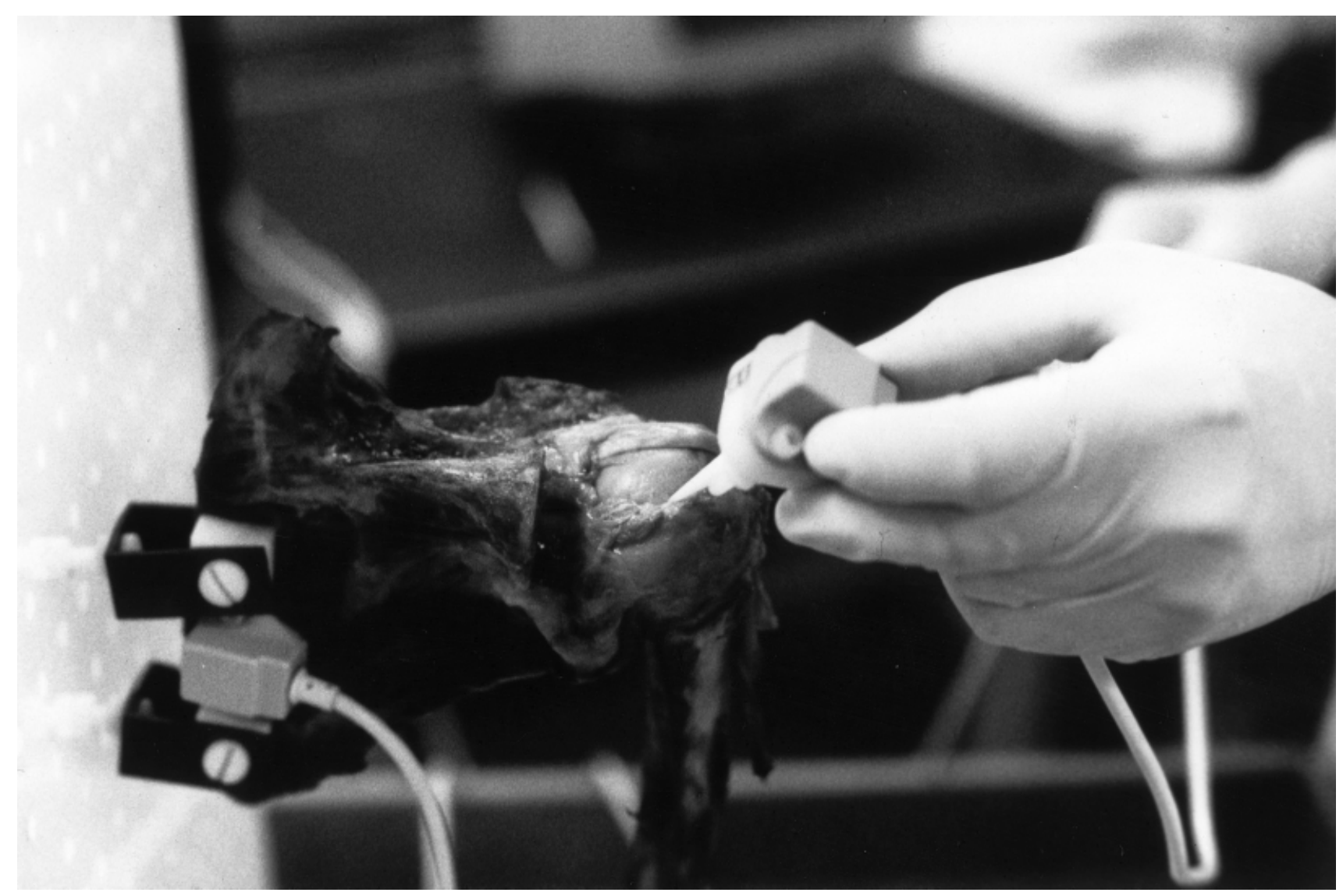

Fig. 1. A point on the border of the RCT being digitized with a probe attached to the base of a Flock of Birds sensor. The medial border of the scapula is affixed to the testing frame. A Flock of Birds sensor is rigidly mounted to the medial rim of the infraspinatus fossa.

nations Program. Since the program provides tissue to multiple institutions, it was impossible to collect the specimens in any patterned or randomized fashion. Institutional Review Board approval was obtained for this study. Of the shoulders collected, 357 specimens, ages 53-86 (mean 70), had no signs of full thickness RCTs. No information regarding the subjects' symptoms was available. Data were collected from the remaining 57 fresh frozen cadaver shoulders, ages 61-94 (mean 78). Non-rotator cuff soft tissue was dissected from each specimen. A blunt dissection of the supraspinatus muscle was preformed so that the digitizing probe could be inserted under the muscle belly to contact the base of the spine of the scapula in the supraspinatus fossa. The medial border of the scapula and the mid-shaft of the humerus were mounted to a testing frame such that the humerus was suspended in a neutral position parallel to the medial border of the scapula in the plane of the scapula. Once the bony landmarks had been digitized, the acromion was removed with a Stryker Bone Saw (Stryker, Kalamazoo, MI) for better access to the RCT.
A Flock of Birds system (Ascension Technologies Corp., Burlington, V'T) was used to digitize the threedimensional coordinates of specific bony and soft tissue landmarks on each specimen. Prior to mounting the specimen, two Flock of Birds sensors were rigidly attached to each specimen using nylon screws. The first sensor was bolted to the medial region of the infraspinatus fossa. The second sensor was bolted to the distal shaft of the humerus. A stylus similar to that described by Meskers et al. (1999) was mounted to a third Flock of Birds sensor to digitize the landmarks and the most internal border of the rotator cuff tear (Fig. 1). Data points circumscribing the RCT were taken at approximately $2 \mathrm{~mm}$ intervals. Prior to beginning each session of digitizing, the workspace was calibrated to account for any variation in the environment, as suggested by Meskers et al. (1999). The same person (R.F.H.) digitized every point on each specimen. The interoperator digitizing error was indistinguishable from the error inherent to the Flock of Birds. Interoperator error was determined by executing the digitization protocol for one specimen four 
times. The variance was then computed for each of the digitized landmarks.

The collected data were converted to a coordinate system referenced to the scapular sensor using software coded in Matlab (MathWorks, Natick, MA). This transformation eliminated the effects of any motion of the specimen on the testing frame during digitizing. The following measurements were computed using software coded in Matlab: the area of the RCT, the tendons included in each tear, the area of the tear in each of the included tendons, the anterior-to-posterior length of the tear at the widest point, and the medialto-lateral length of the tear at the widest point.

The area of the RCT was defined as the area inside a two-dimensional polygon described by the perpendicular projection of each of the RCT data points onto a plane. The plane of the tear was determined by taking the planar least-squares fit (Van der Helm et al., 1992) of the data points circumscribing the RCT. Two tendon separation planes were then used to locate the border of the included tendons: one plane represented the border between the infraspinatus and supraspinatus and the second represented the border between the supraspinatus and the subscapularis. The infraspinatus-supraspinatus separation plane was defined by points digitized at three scapular landmarks: medial border at the intersection of the scapular spine, posterolateral acromion, and a point at the base of the spine of the scapula in the supraspinatus fossa. The supraspinatus-subscapularis separation plane was determined in a like manner, using points digitized at the superior angle, scapular notch, and superior anterior corner of the coracoid. The RCT data points were then segregated into their respective tendons such that any point posterior to the infraspinatus-supraspinatus plane belonged to the infraspinatus, any point between the two planes belonged to the supraspinatus, and any point anterior to the supraspinatus-subscapularis plane belonged to the subscapularis. The area of the tear in a given tendon was computed in a similar fashion to that used for the entire tear. The points where the tear intersected a separation plane were included in the segregated datasets to ensure that none of the area of the tear was lost in the process of distributing the data points to their respective tendons.

For tears including the supraspinatus tendon, the dimension of the tear in the medial-to-lateral direction and anterior-to-posterior direction were computed at the widest point. A line in the anterior-posterior direction was determined by the following algorithm: the lines describing the intersection of the plane of the supraspinatus tear and the planes defining the tendon borders were established; a line in the plane of

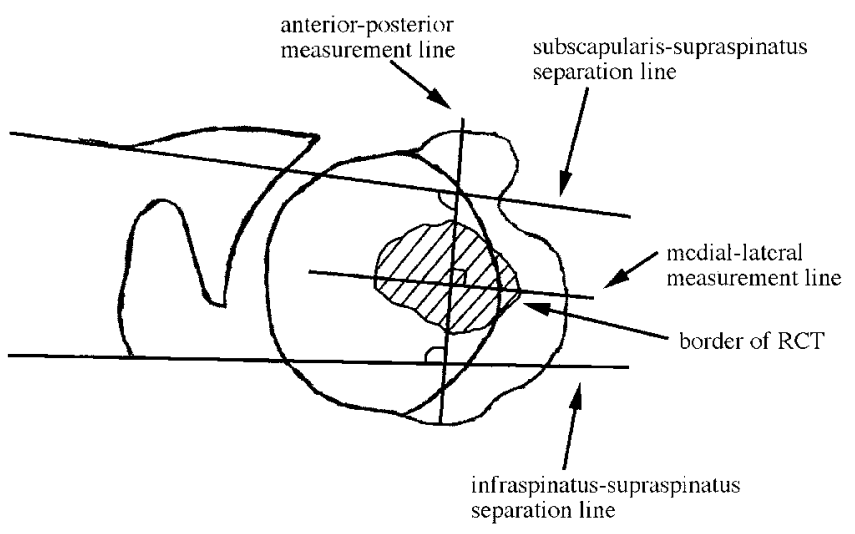

Fig. 2. A superior view of the shoulder with the acromion removed to show an RCT in the lateral region of the humeral head. A line intersecting the infraspinatus-supraspinatus separation line and the supraspinatus-subscapularis separation line is used to measure the tear in the anterior-posterior direction. A line perpendicular to the anterior-posterior line is used to measure the tear in the medial-lateral direction. Measurements are taken at the widest point of the tear.

the tear in the anterior-posterior direction was chosen such that it intersected both of these lines at equal angles (Fig. 2). The length of the tear in the anteriorposterior direction was calculated along this line at the widest portion of the tear. The line in the mediallateral direction was chosen as the line in the plane of the tear perpendicular to the anterior-posterior line. The length of the tear in the medial-lateral direction was calculated along this line at the widest portion of the tear.

\section{RESULTS}

Of the 414 shoulders collected during the course of this study, 57 (16\%) had full thickness RCTs. Of the normal shoulders, 184 were female and 173 were male. Of the shoulders with RCTs, 37 were female, 20 male, 25 were left arms, and 32 were right arms. The shoulders came from 38 cadavers, 23 female and 15 male. Nineteen of the cadavers had bilateral RCTs while the remaining 19 possessed unilateral RCTs. No correlation between area of RCT and age was found $(P>0.564)$.

Of the 57 specimens collected with full thickness RCTs, six had tears only in the infraspinatus, 25 only in the supraspinatus, one only in the subscapularis, 22 in the infraspinatus and supraspinatus, one in the supraspinatus and subscapularis, and two had a tear including all three of the major tendons (Table 1).

The mean, minimum, maximum, and median calculated areas of the RCT are listed in Table 2 by the included tendons. The overall largest tear included the supraspinatus and the infraspinatus with a size of $19.17 \mathrm{~cm}^{2}$. The smallest tear was contained within the 
TABLE 1. Tendons Studied

\begin{tabular}{lccccccc}
\hline Tendons included in tear & Inf & Sup & Sub & Inf + Sup & Sup + Sub & All tendons & All specimens \\
\hline Percent of all shoulders & 1.68 & 7 & 0.28 & 6.16 & 0.28 & 0.56 \\
Number with tear & 6 & 25 & 1 & 22 & 1 & 2 & 15.96 \\
Percent of shoulders with full thickness RCTs & 10.53 & 43.86 & 1.75 & 38.6 & 1.75 & 3.51 & 100 \\
\hline
\end{tabular}

For each combination of tendons, the percentage of the total shoulders collected with that type of tear, the number with that type of tear, and the percentage of shoulders with a tear having that type of tear are listed. Infraspinatus (Inf), Supraspinatus (Sup), Subscapularis (Sub).

supraspinatus and was $0.07 \mathrm{~cm}^{2}$. The overall mean tear size was $4.40 \mathrm{~cm}^{2}$. The anterior-posterior length measurement ranged from $0.20-6.00 \mathrm{~cm}$ with a mean of $1.95 \mathrm{~cm}$ and a median of $1.60 \mathrm{~cm}$. The mediallateral measurement ranged from $0.30-5.31 \mathrm{~cm}$ with a mean of $1.98 \mathrm{~cm}$ and a median of $1.38 \mathrm{~cm}$.

\section{DISCUSSION}

In this study the prevalence of full thickness RCTs at the time of death was found to be $16 \%$. A quantitative description of the area of those full thickness tears, and the distribution of those tears among the tendons of the rotator cuff, was also determined. For any tear including the supraspinatus tendon, anteriorposterior and medial-lateral dimensions of the tear were calculated.

The percentage of shoulders with full thickness RCTs in this population was found to be similar to the percentages currently reported in the literature ('Table $3)$. Of these studies only one categorized the tear based on the individual tendons of the rotator cuff (Itoi et al., 1995). Itoi et al. (1995) studied differences between the extramuscular tendon length and the functional tendon length to determine the size of the RCT but did not specifically report tear sizes. Sher et al. (1995) noted tear sizes ranging from $0.5-14 \mathrm{~cm}^{2}$ (mean $5.0 \pm 4.3 \mathrm{~cm}^{2}$ ) based on MR images of asymptomatic individuals. A description of the measurement technique is not provided. It should be noted that the mean and standard deviation for this study and the Sher et al. (1995) study are very similar. A cadaver study comparing the sizes of RCTs found using MR images to those found via dissection and digitizing with the Flock of Birds system may be an effective method for validating the accuracy of MR images for measuring RCT areas.

The correlation between increased age and prevalence of RCTs is well documented (Keyes, 1933; Ogata and Uhthoff, 1990; Sher et al., 1995; Tempelhof et al., 1999; Wilson and Duff, 1943; Zuckerman et al., 1992). In this study it was also noted that the prevalence of RCTs increased as age increased. A correlation between the area of the RCT and the age of the subject was not found $(P>0.564)$. This is not entirely surprising, since the date of onset and cause (traumatic, nontraumatic) of the lesion was unknown.

Due to the nature of the data collection and processing, it is possible that the areas reported for the RCTs in this study are slightly smaller than the true area. The digitizer was consistently placed such that the probe tip was inside the tear and lightly contacting the tear. The area reported is the planar projection of the data points defining the border of the RCT, and therefore does not account for the cylindrical nature of the capsule. However, it should be noted that the errors in measurement produced by this planar projection are negligibly small for small tears and only of minor concern for the largest tears reported in this study.

In this study the supraspinatus was clearly the most commonly affected tendon, while RCTs affecting both the supraspinatus and infraspinatus followed closely. Involvement of the subscapularis was rare. The rotator interval between the supraspinatus and the subscapularis contains the biceps tendon. Although this region of the capsule is relatively thin, the sheath surrounding the biceps is reinforced by fibers

TABLE 2. Smallest, Largest, Mean, and Median Tear Size for Each Combination of Tendons Torn

\begin{tabular}{|c|c|c|c|c|c|c|c|}
\hline Tendons included in tear & $\begin{array}{l}\operatorname{Inf} \\
\mathrm{cm}^{2}\end{array}$ & $\begin{array}{l}\text { Sup } \\
\mathrm{cm}^{2}\end{array}$ & $\begin{array}{l}\text { Sub } \\
\mathrm{cm}^{2}\end{array}$ & $\begin{array}{c}\text { Inf }+ \text { Sup } \\
\mathrm{cm}^{2}\end{array}$ & $\begin{array}{c}\text { Sup } \\
\mathrm{cm}^{2}\end{array}$ & $\begin{array}{l}\text { All tendons } \\
\mathrm{cm}^{2}\end{array}$ & $\begin{array}{c}\text { All specimens } \\
\mathrm{cm}^{2}\end{array}$ \\
\hline Number with tear & 6 & 25 & 1 & 22 & 1 & 2 & 57 \\
\hline Smallest tear & 0.16 & 0.07 & 0.25 & 0.22 & 7.09 & 9.85 & 0.07 \\
\hline Largest tear & 3.97 & 12.16 & 0.25 & 19.17 & 7.09 & 18.60 & 19.17 \\
\hline Mean tear & 1.41 & 2.38 & 0.25 & 6.77 & 7.09 & 14.23 & 4.43 \\
\hline Median tear & 0.69 & 0.65 & 0.25 & 4.20 & 7.09 & 14.23 & 2.40 \\
\hline
\end{tabular}

Infraspinatus (Inf), Supraspinatus (Sup), Subscapularis (Sub). 
TABLE 3. Results of Previous Studies

\begin{tabular}{|c|c|c|c|c|c|}
\hline Study & Number of specimens & $\begin{array}{l}\text { Age range } \\
(\text { mean })\end{array}$ & $\begin{array}{c}\text { Sex } \\
(\mathrm{M} / \mathrm{F})\end{array}$ & $\begin{array}{c}\text { Number of specimens } \\
\text { with RCTs }\end{array}$ & $\begin{array}{c}\text { Percent of specimens } \\
\text { with RCTs }\end{array}$ \\
\hline Yamanaka et al., 1983 & 249 & N/A & $\mathrm{N} / \mathrm{A}$ & 18 & 7 \\
\hline Hijioka et al., 1993 & 160 & $43-94$ & $112 / 48$ & 18 & 11.3 \\
\hline Keyes, 1933 & 146 & N/A & N/A & 19 & 13.1 \\
\hline Sher et al., 1995 & 96 & $19-88(53)$ & $47 / 49$ & 14 & 15 \\
\hline Wilson and Duff, 1943 & 148 & $\mathrm{~N} / \mathrm{A}$ & N/A & 22 & 15 \\
\hline This Study & 414 & $53-94(71)$ & $193 / 224$ & 57 & 15.9 \\
\hline Lehman et al., 1995 & 456 & $27-102$ & $298 / 256$ & 77 & 17 \\
\hline Wilson and Duff, 1943 & 68 & N/A & N/A & 14 & 21 \\
\hline Templehof et al., 1999 & 411 & 60 -over 80 & N/A & 95 & 23 \\
\hline Bigliani et al., 1986 & 142 & $51-97$ & $74 / 68$ & 34 & 24 \\
\hline Ogata and Uhthoff, 1990 & 76 & $34-87$ (69) & $32 / 44$ & 19 & 25 \\
\hline Itoi et al., 1995 & 41 & $64-96(84)$ & N/A & 18 & 44 \\
\hline
\end{tabular}

Studies are rank ordered by percentage of specimens with RCTs.

of the coracohumeral ligament. This reinforcement may serve as a buttress to prevent anterior extension of supraspinatus tears. Clearly, more research is needed to understand the mechanism behind the extension of RCTs.

\section{ACKNOWLEDGMENTS}

The authors thank Laurie Huston, Linda Gallo, Mark Stock, and the Department of Defense Science and Engineering Graduate Fellowship.

\section{REFERENCES}

Bigliani LU, Morrison DS, April EW. 1986. The morphology of the acromion and its relationship to rotator cuff tears. Orthop Trans 10:228.

Chang Y-W, Hughes RE, Su F-C, Itoi E, An K-N. 2000. Prediction of muscle force involved in shoulder internal rotation. J Shoulder Elbow Surg 9:188-195.

Hijioka A, Suzuki K, Nakamura T, Hojo T. 1993. Degenerative change and rotator cuff tears. Arch Orthop Trauma Surg 112:61-64.

Hughes RE, An K-N. 1997. Monte Carlo simulation of a planar shoulder model. Med Biol Eng Comput 35:544-548.

Hughes RE, Rock MG, An K-N. 1999. Identification of optimal strategies for increasing whole arm strength using KarushKuhn-Tucker multipliers. Clin Biomech 14:628-634.

Itoi E, Hsu HC, Carmichael SW, Morrey BF, An KN. 1995. Morphology of the torn rotator cuff. J Anat 86:429-434.
Keyes EL. 1933. Observations on rupture of the supraspinatus muscle. Ann Surg 97:849-856.

Lehman C, Cuomo F, Kummer FJ, Zuckerman JD. 1995. The incidence of full thickness rotator cuff tears in a large cadaveric population. Hosp Joint Dis 54:30-31.

Meskers CGM, Fraterman H, van der Helm FCT, Vermeulen HM, Rozing PM. 1999. Calibration of the "Flock of Birds" electromagnetic tracking device and its application in shoulder motion studies. J Biomech 32:629-633.

Ogata S, Uhthoff HK. 1990. Acromial enthesopathy and rotator cuff tear: a radiologic and histologic postmortem investigation of the coracoacromial arch. Clin Orthop 254:39-48.

Sher JS, Uribe JW, Posada A, Murphy BJ, Zlatkin MB. 1995. Abnormal findings on magnetic resonance images of asymptomatic shoulders. J Bone Joint Surg 77A:10-15.

Tempelhof S, Rupp S, Seil R. 1999. Age-related prevalence of rotator cuff tears in asymptomatic shoulders. J Shoulder Elbow Surg 8:296-299.

Van der Helm FCT, Veeger HEJ, Pronk GM, Van der Woude LHV, Rozendal RH. 1992. Geometry parameters for musculoskeletal modeling of the shoulder system. J Biomech 25:129-144.

Wilson CL, Duff GL. 1943. Pathologic study of degeneration and rupture of the supraspinatus tendon. Arch Surg 47:121135.

Yamanaka K, Fukuda H, Hamada K, Mikasa M. 1983. Partial thickness tear of the rotator cuff. Rinsho Seikeigeka 26:713723.

Zuckerman JD, Kummer FJ, Cuomo F, Simon J, Rosenblum S, Katz N. 1992. The influence of coracoacromial arch anatomy on rotator cuff tears. J Shoulder Elbow Surg 1:4-14. 